\title{
Accuracy of Endoscopic Diagnosis of Mild Atrophic Gastritis with Helicobacter pylori Infection
}

\author{
Dae Bum Kim and Woo Chul Chung \\ Department of Internal Medicine, St. Vincent's Hospital, College of Medicine, The Catholic University of Korea, Suwon, Korea
}

See "Accuracy of Endoscopic Diagnosis for Mild Atrophic Gastritis Infected with Helicobacter pylori" by Takuma Okamura, Yugo Iwaya, Kei Kitahara et al., on page 362-367.

Helicobacter pylori infection is responsible for chronic gastritis. The progression from chronic gastritis, atrophy, and intestinal metaplasia to dysplasia is an important mechanism of gastric carcinogenesis. A meta-analysis showed that $H$. $p y$ lori eradication reduced the risk of the development of gastric cancer. ${ }^{1}$ However, a later study showed that the risk of gastric cancer was still increased in the presence of gastric mucosal atrophy, even after $H$. pylori infection was eradicated. ${ }^{2}$ These findings may imply that gastric cancer is entirely preventable by $H$. pylori eradication in patients with atrophic gastritis. On the basis of previous evidence, we surmise that eradication therapy for $H$. pylori infection could be more efficient in preventing gastric cancer when mucosal atrophy is mild or absent. It is likely that the progression to gastric cancer can be blocked before gastric atrophy develops. In other words, endoscopists should be aware that the identification of mild atrophic gastritis associated with $H$. pylori infection is important in the primary prevention of gastric cancer. This is especially important in the training of beginner endoscopists.

This study showed that the accuracy of endoscopic diagno-

Received: May 23, 2018 Revised: May 29, 2018 Accepted: May 29, 2018

Correspondence: Woo Chul Chung

Division of Gastroenterology, Department of Internal Medicine, St. Vincent's Hospital, College of Medicine, The Catholic University of Korea, 93 Jungbu-daero, Paldal-gu, Suwon 16247, Korea

Tel: +82-31-249-7138, Fax: +82-31-253-8898, E-mail: jwchulkr@catholic.ac.kr ORCID: https://orcid.org/0000-0003-1044-0440

cc This is an Open Access article distributed under the terms of the Creative Commons Attribution Non-Commercial License (http://creativecommons.org/ licenses/by-nc/3.0) which permits unrestricted non-commercial use, distribution, and reproduction in any medium, provided the original work is properly cited. sis of $H$. pylori infection in patients with/without mild atrophic gastritis (C-0, C-I) was approximately $67 \% .^{3}$ In previous studies, the following distinctive endoscopic features were reported and were closely associated with $H$. pylori infection: atrophy, hyperplastic polyp, map-like/patchy/spotty redness, xanthoma, hematin, red streak, intestinal metaplasia, mucosal swelling, depressed erosion, enlarged fold, sticky mucus, fundic gland polyp, multiple white and flat elevated lesions, correctly identified regular arrangement of collecting venules, nodularity, and raised erosion. ${ }^{4-8}$ Among these endoscopic features, nodularity had the highest diagnostic accuracy for $H$. pylori infection (89.3\%), followed by mucosal swelling (76.6\%). When endoscopists identified nodularity or mucosal swelling, the rate of diagnostic accuracy was high (Fig. 1). The characteristic histological finding of nodularity is hyperplasia of lymphoid follicles, whereas mucosal swelling is characterized by inflammatory cell infiltration and mucosal edema. In the progression of atrophy, nodularity begins to disappear from the antrum and becomes more localized with scattered type. Therefore, endoscopists should search carefully for characteristic nodularity, especially at the gastric angle and anterior/ posterior walls of the antrum. Mucosal swelling is easier to recognize in the gastric body than in the antrum. Close attention to nodularity and mucosal swelling may improve the accuracy of endoscopic diagnosis of $H$. pylori infection in mild atrophic gastritis.

Although a number of studies have discussed endoscopic diagnosis of $H$. pylori infection, the findings have been ambiguous and contradictory. It was previously reported that the accuracy of endoscopic diagnosis of $H$. pylori infection was 

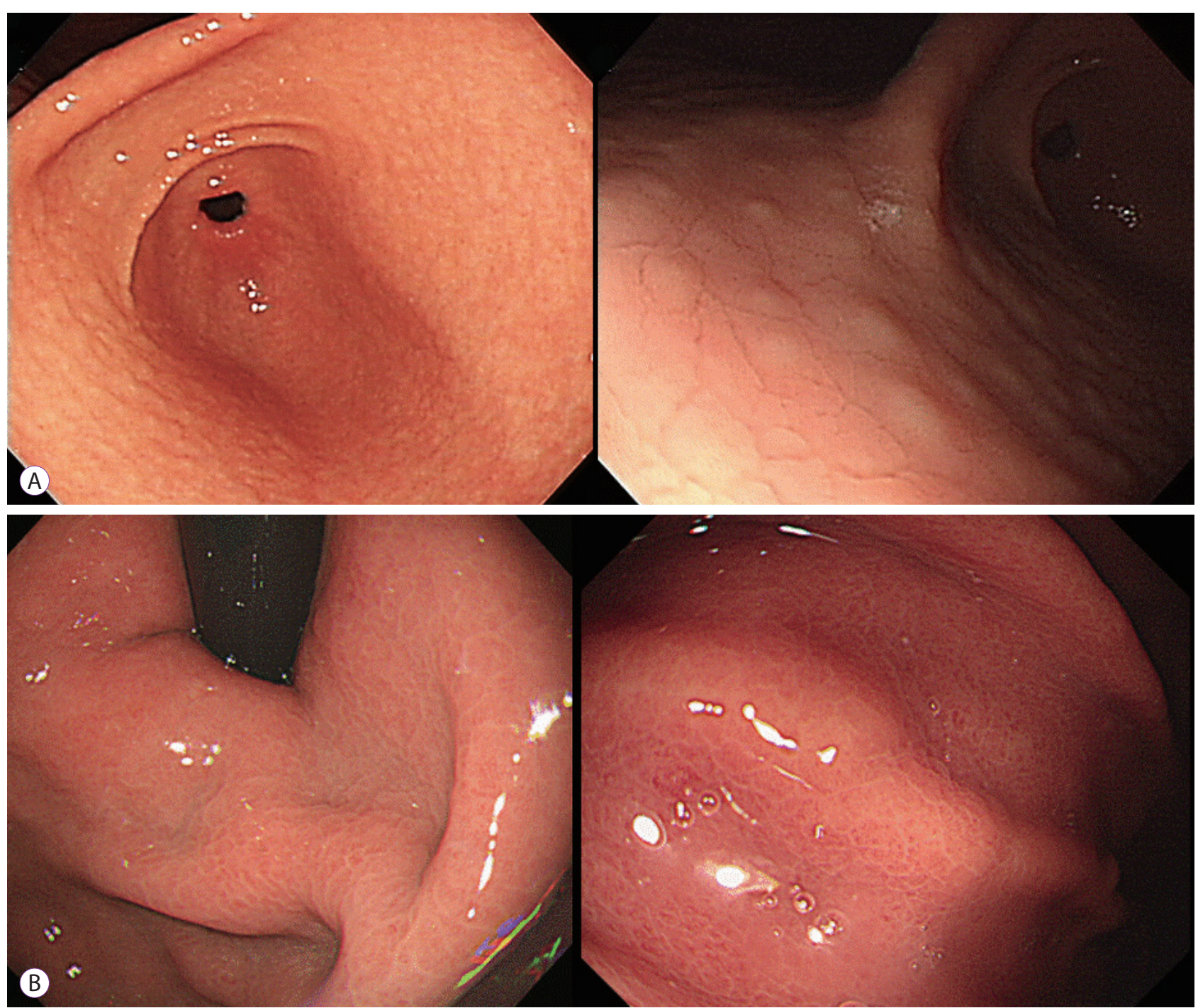

Fig. 1. Endoscopic findings of nodularity or mucosal swelling, which are closely related to the early stage Helicobacter pylori gastritis. (A) Nodularity. (B) Mucosal swelling.

only $41.8 \%{ }^{9}$ In a series of studies, several researchers insisted that endoscopic diagnosis of $H$. pylori infection was of no use in clinical practice, because of relatively low diagnostic yield $(43 \%-64 \%)^{10,11}$ On the other hand, a study in highly-endemic areas of $H$. pylori infection revealed that the accuracy of endoscopic diagnosis reached $79.5 \% .{ }^{12}$ Taken together, the inconsistency of diagnostic yields might stem from differences in regional prevalence of $H$. pylori infection. The results of this study were quite acceptable, although the diagnostic yield was relatively low compared with that of previous studies. The reason was that the study was performed only in cases of mild or non-atrophic gastritis. In previous reports, regular arrangement of collecting venules, hemorrhage, and fundic gland polyps showed high positive predictive value and specificity for an $\mathrm{H}$. pylori-negative stomach. ${ }^{6,13-15}$ Moreover, the diagnostic yield in $H$. pylori-negative cases was high, and in- ter-observer agreement was good. In the wake of this research, there are several problems to be solved. First, as for nodularity and mucosal swelling, inter- and intra-observer agreement is questionable. Second, it is necessary to confirm whether endoscopists can distinguish between $H$. pylori infection and $H$. pylori eradication.

Recently, artificial intelligence technology was evaluated in the diagnosis of $H$. pylori gastritis based on endoscopic images, and was able to diagnose $H$. pylori gastritis with higher accuracy. ${ }^{16}$ It also shortened the diagnostic time, compared with manual diagnosis by an endoscopist. Endoscopic diagnosis using artificial intelligence has an infinite potential, and it will be supplemented and completed by the accumulation of these findings.

In conclusion, early detection of $H$. pylori gastritis and prompt eradication is a good therapeutic strategy for the pre- 
vention of gastric cancer. From this point of a view, this result is promising and will contribute to the early detection of $H$. pylori gastritis.

\section{Conflicts of Interest}

The authors have no financial conflicts of interest.

\section{REFERENCES}

1. Fuccio L, Zagari RM, Eusebi LH, et al. Meta-analysis: can Helicobacter pylori eradication treatment reduce the risk for gastric cancer? Ann Intern Med 2009;151:121-128.

2. Take S, Mizuno M, Ishiki K, et al. The long-term risk of gastric cancer after the successful eradication of Helicobacter pylori. J Gastroenterol 2011;46:318-324

3. Okamura T, Iwaya Y, Kitahara K, Suga T, Tanaka E. Accuracy of endoscopic diagnosis for mild atrophic gastritis infected with Helicobacter pylori. Clin Endosc 2018;51:362-367.

4. Kato T, Yagi N, Kamada T, Shimbo T, Watanabe H, Ida K. Diagnosis of Helicobacter pylori infection in gastric mucosa by endoscopic features: a multicenter prospective study. Dig Endosc 2013;25:508-518.

5. Watanabe K, Nagata N, Nakashima R, et al. Predictive findings for Helicobacter pylori-uninfected, -infected and -eradicated gastric mucosa: validation study. World J Gastroenterol 2013;19:4374-4379.

6. Yagi K, Nakamura A, Sekine A. Characteristic endoscopic and magnified endoscopic findings in the normal stomach without Helicobacter pylori infection. J Gastroenterol Hepatol 2002;17:39-45.

7. Mao T, Wang Y, Yin F, et al. Association of endoscopic features of gastric mucosa with Helicobacter pylori infection in Chinese patients. Gastroenterol Res Pract 2016;2016:6539639.

8. Bah A, Saraga E, Armstrong D, et al. Endoscopic features of Helicobacter pylori-related gastritis. Endoscopy 1995;27:593-596.

9. Khakoo SI, Lobo AJ, Shepherd NA, Wilkinson SP. Histological assessment of the Sydney classification of endoscopic gastritis. Gut 1994;35:1172-1175.

10. Belair PA, Metz DC, Faigel DO, Furth EE. Receiver operator characteristic analysis of endoscopy as a test for gastritis. Dig Dis Sci 1997;42:22272233.

11. Redeen S, Petersson F, Jonsson KA, Borch K. Relationship of gastroscopic features to histological findings in gastritis and Helicobacter pylori infection in a general population sample. Endoscopy 2003;35:946-950.

12. Mihara M, Haruma K, Kamada T, et al. The role of endoscopic findings for the diagnosis of Helicobacter pylori infection: evaluation in a country with high prevalence of atrophic gastritis. Helicobacter 1999;4:40-48.

13. Kaminishi M, Yamaguchi H, Nomura S, et al. Endoscopic classification of chronic gastritis based on a pilot study by the research society for gastritis. Dig Endosc 2002;14:138-151.

14. Watanabe N, Seno H, Nakajima T, et al. Regression of fundic gland polyps following acquisition of Helicobacter pylori. Gut 2002;51:742-745.

15. Na S, Chung JW, Park HJ, et al. [The usefulness of the regular arrangement of collecting venules pattern for the determination of Helicobacter pylori infection]. Korean J Gastroenterol 2011;58:252-257.

16. Shichijo S, Nomura S, Aoyama K, et al. Application of convolutional neural networks in the diagnosis of Helicobacter pylori infection based on endoscopic images. EBioMedicine 2017;25:106-111 